\title{
Fake brain helping human brain
}

\section{Artificial intelligence in mental health care}

Anastasiya Vinokurtseva, Shafaz Veettil

\begin{abstract}
Mental health disorders affect $30 \%$ of the world's population and rates of diagnosis continue to increase. This huge global burden on the individual and societal levels requires enhancement of current diagnosis and treatment. Artificial intelligence (AI) offers novel approaches to diagnosis in mental health care. Personal digital devices that have become omnipresent in the developed world can be used as sensors for continuous monitoring of an individual's behaviour. Analysis of behavioural markers such as sleep, social media use, and communication patterns through the use of sensors creates a patient's digital phenotype. Continuous monitoring offers an insight into patients' regular behaviour that is different from the self-reported, momentary snapshot used in conventional diagnostic practices. Virtual humans offer an alternative way to collect information, allowing for increased patient disclosure. Current applications of AI facilitate access to mental health care by monitoring patient functioning in real life and immediately connecting patients to appropriate resources. Despite many benefits of AI in mental health care, further translation of this innovation from research to clinical practice requires diligent policy making to address privacy and confidentiality concerns.
\end{abstract}

\section{INTRODUCTION}

Increased focus on mental health disorders has allowed for a more open dialogue regarding their prevalence and a more precise estimate of their epidemiological significance. ${ }^{1}$ Various international studies estimate that a third of the world's population is affected by mental disorders, and the rates of diagnosis continue to increase. ${ }^{1,2}$ Global burden is aggravated by high co-morbidity, as several mental disorders often occur together. ${ }^{3}$ Cost to individuals and society is immense, as mental disorders negatively affect quality of life, economy, and longevity. ${ }^{4,5}$ Conventional diagnosis of mental health usually depends on the health care provider's interpretation of patient-reported signs and symptoms with respect to diagnostic criteria and classification systems. ${ }^{6,7}$ However, the process has several limitations, such as patients' lack of disclosure, providers' inadequate recognition of variable disease presentations, and systemic constraints including time and productivity pressures. ${ }^{78}$ A solution to many of these challenges could lie in the use of artificial intelligence (AI), whereby various devices collect and analyze patient data, and the process of machine learning (ML) develops appropriate algorithms to recognize and treat mental disorders. ${ }^{8,9}$ This review will discuss applications of this concept in mental health care, as well as advantages, barriers and important considerations with respect to increased digitalization of care.

\section{ARTIFICIAL INTELLIGENCE AND MENTAL HEALTH}

Considering that psychiatric disorders have a diverse variety of presentations, one way to improve diagnosis and treatment is access to a more personal and continuous monitoring of a patient's status. ${ }^{7,8}$ Potential sensors for collection of a wide array of behavioural information include smartphones, smartwatches, computers, social media, and online browsing, which have become omnipresent in the developed world..$^{8,10}$ Patients, consciously or not, are constantly inputting data into these devices, which could be used for mental health diagnoses. 11,12 Such data includes communication type, frequency and duration of communications, hobbies and interests, sleep patterns, and physical activity. ${ }^{11,12}$

Using accelerometry, wearable devices and smartphones allow for continuous monitoring of physical activity, which is both an important diagnostic marker and treatment modality in mental health. ${ }^{11-13}$ Online browsing reveals personal hobbies and interests, while social media posts contain verbal cues related to mental health diagnoses. ${ }^{11,14}$ Sleep patterns can be monitored through screen lock times and battery usage. ${ }^{11}$ Finally, communication frequency, timing, duration, and type (via social media, text messages, or phone calls) may be indicative of relationships and support networks present in the individual's life. ${ }^{11}$

Collection of these diagnostic behavioural markers allows for digital phenotyping-continuous quantification of an individual's phenotype using digital devices. ${ }^{12}$ Digital phenotyping is a useful tool for determination of the patient's baseline functioning in real life, which may be different from that observed in a health care setting. Additionally, it allows for real-time monitoring of any changes to determine risk factors and offer appropriate interventions. ${ }^{12,15}$

Another innovation in mental health diagnosis involves the use of virtual humans to collect patient data. ${ }^{16,17}$ While conventional interviews in health care setting are conducted by a human, advances in technology can replace the interviewer with an interactive $3 \mathrm{D}$ projection of a person on the screen, who can be fully automated to conduct a semi-structured interview. ${ }^{16,17} \mathrm{ML}$ can analyse linguistic and paralinguistic cues in the patient's response and direct the conversation accordingly. ${ }^{11,17}$ Besides collecting verbal input, the virtual human also recognizes non-verbal cues, analyzes the patient's emotions and responds appropriately, helping to establish rapport. ${ }^{17}$ Studies have found that this advance in AI facilitates patients' disclosure by reducing perceived judgement and fear of negative evaluation, as well as increasing the sentiment of privacy. ${ }^{16-19}$ 


\section{IMPLICATIONS TO PRACTICE}

In comparison with conventional practices, AI applications offer insight into the daily lives of patients, which is different from what a health care provider can observe in a health care setting. ${ }^{8,11,12}$ AI analyzes a variety of data input to create an individual digital phenotype. ${ }^{12}$ Some of the aggregated behavioural markers are important in conventional mental health diagnostics. ${ }^{10}$ For instance, sleep disruptions and reduced social contact have been linked to depression. ${ }^{10,11,20}$ In conventional practice, this type of data would be self-reported and could be skewed by an individual's perception, but digital devices allow for thorough and continuous monitoring and evaluation of these markers. ${ }^{10,11}$ Similarly, conventional diagnostic markers, such as linguistic cues, can now be observed in various social contexts. ${ }^{8,11,12}$ Social media posts can be monitored for manifestations of hopelessness, increased self-attention, and reduced coordination with the community, which indicate patterns of depression and suicidal tendencies. ${ }^{11,20} \mathrm{ML}$ and predictive modeling can foresee depressive episodes and allow for timely interventions. ${ }^{8,21}$

Besides conventional diagnostic markers, the advent of technological innovations permits the use of behavioural markers which are difficult to perceive, quantify, and analyze using conventional practices. ${ }^{11,12,22}$ An example of such markers are paralinguistic cues such as vocal pitch, rate, inflection, and tone. ${ }^{11,12,16,22}$ These cues are part of conventional interviews but a practitioner may interpret these subtle metrics in a more subjective way than AI, which utilizes more rigorous voice analytics. ${ }^{11,16}$

Technological advances facilitate access to health care by automating the process of data collection that can be used in diagnosis, and connecting the person to the appropriate preventative and treatment services. ${ }^{23,24}$ This is particularly important considering the constraints of our medical system. ${ }^{23} \mathrm{An}$ example of current applications of AI in psychiatric care involves Tess the chatbot.9,24 The chatbot works as a personal coach, helping people work through difficult situations using cognitive behavioral therapy. ${ }^{24,25}$ If Tess recognizes any risk factors in a patient's text messages, it immediately communicates this to a therapist, who is then able to intervene appropriately. ${ }^{24}$ In traditional access to care, patients need to endure long waitlists before accessing any screening or diagnostic services. ${ }^{12}$ During this time, their condition could advance to require more complex interventions. ${ }^{12}$ AI streamlines the diagnostic process, offers immediate interventions, and connects the patient to professional help if needed. ${ }^{12,24}$ Such an application of AI facilitates access to care and optimizes health care delivery. ${ }^{23-25}$

\section{FURTHER CONSIDERATIONS}

While AI and ML offer significant benefits in the diagnosis and management of mental health, there are some concerns associated with this innovation. ${ }^{10}$ Although studies have found an increased sentiment of privacy and patient disclosure during interviews with virtual humans, it may be challenging to overcome privacy concerns when it comes to AI analysis of smartphone and social media use..$^{10,11,16}$ Considering the breadth of data that may be accessible from such devices, patients may be hesitant to have this data analyzed by the machines and linked to their health status..$^{10,15,26}$ As with many innovations, the concern becomes when, how, and who will have access to this information. ${ }^{27}$ Rigorous control and consent measures will be necessary to ensure patient privacy and confidentiality in the translation of these innovations from research to clinical practice. ${ }^{27,28}$ On the other side, marketing companies already undertake a significant analysis of the population's digital device use. ${ }^{26}$ Potentially, a significant part of that data would overlap with health-related data, and the balance between these datasets would be an interesting area of investigation and an important factor in policy making on the subject. ${ }^{26-28}$

\section{CONCLUSION}

The global burden of mental health disorders is high: they are estimated to affect a third of the world's population, with rates of diagnosis continuously rising., ${ }^{1,2}$ These conditions have significant implications on individuals and on Canadian society, affecting daily functioning and quality of life, and costing Can $\$ 51$ billion per year to the Canadian economy. ${ }^{4,29}$ AI applications in mental health care could facilitate and expedite access to care, allow for early diagnosis, and promote timely interventions. ${ }^{8,11,12}$ AI allows for more continuous monitoring of patient status, but raises some privacy concerns which would need to be addressed by policymakers in the process of translation of this technology from research to clinical practice. .11,26,27 $^{2}$

\section{REFERENCES}

1. Polanczyk GV, Salum GA, Sugaya LS, et al. Annual research review: a meta-analysis of the worldwide prevalence of mental disorders in children and adolescents. J Child Psychol Psychiatry Allied Discip. 2015 Mar;56(3):345-65. https://doi.org/10.1111/jcpp.12381

2. Steel Z, Marnane C, Iranpour C, et al. The global prevalence of common mental disorders: a systematic review and meta-analysis 1980-2013. Int J Epidemiol. 2014 Apr;43(2):476-93. https://doi. org/10.1093/ije/dyu038

3. Kessler RC, Birnbaum HG, Shahly V, et al. Age differences in the prevalence and comorbidity from the WHO World Mental Health Survey Initiative. Depress Anxiety. 2010 Apr;27(4):351-64. https://doi. org/10.1002/da.20634

4. Whiteford HA, Degenhardt L, Rehm J, et al. Global burden of disease attributable to mental and substance use disorders: findings from the Global Burden of Disease Study 2010. Lancet. 2013 Nov;382(9904):1575-86. https://doi.org/10.1016/S0140-6736(13)61611-6

5. Davlasheridze M, Goetz SJ, Han Y. The effect of mental health on U.S. county economic growth. Rev Reg Stud. 2018 Jan;48(2):155-71.

6. Mitchell AJ, Vaze A, Rao S. Clinical diagnosis of depression in primary care: a meta-analysis. Lancet. 2009 Aug;374(9690):609-19. https://doi. org/10.1016/S0140-6736(09)60879-5

7. Cepoiu M, McCusker J, Cole MG, et al. Recognition of depression by non-psychiatric physicians - a systematic literature review and meta-analysis. J Gen Intern Med. 2008 Jan;23(1):25-36. https://doi. org/10.1007/s11606-007-0428-5

8. Akeret K, Vasella F, Geisseler O, et al. Time to be "smart "opportunities arising from smartphone-based behavioral analysis in daily patient care. Front Behav Neurosci. 2018 Dec;12:10-3. https://doi. 
org/10.3389/fnbeh.2018.00303

9. Tiffin PA, Paton LW. Rise of the machines? Machine learning approaches and mental health: opportunities and challenges. Br J Psychiatry. 2018 Sept;213(3):509-10. https://doi.org/10.1192/ bjp.2018.105

10. Torous J, Larsen ME, Depp C, et al. Smartphones, sensors, and machine learning to advance real-time prediction and interventions for suicide prevention: a review of current progress and next steps. Curr Psychiatry Rep. 2018 Jun;20:51. https://doi.org/10.1007/s11920018-0914-y

11. Mohr DC, Zhang M, Schueller SM. Personal sensing: understanding mental health using ubiquitous sensors and machine learning. Annu Rev Clin Psychol. 2017 May;13:23-47 https://doi.org/10.1146/annurevclinpsy-032816-044949

12. Sano A, Taylor S, McHill AW, et al. Identifying objective physiological markers and modifiable behaviors for self-reported stress and mental health status using wearable sensors and mobile phones: observational study. J Med Internet Res. 2018 Jun;20(6):e210. https:// doi.org/10.2196/jmir.9410

13. Naslund JA, Aschbrenner KA, Bartels SJ. Wearable devices and smartphones for activity tracking among people with serious mental illness. Ment Heal Phys Act. 2016 Mar;10:10-7. https://doi. org/10.1016/i.mhpa.2016.02.001

14. Frison E, Eggermont S. Browsing, posting, and liking on Instagram: the reciprocal relationship between different types of Instagram use and adolescents' depressed mood. Cyberpsychol Behav Soc Netw. 2017 Oct;20(10):603-9. https://doi.org/10.1089/cyber.2017.0156

15. Hoffman L, Benedetto E, Huang $\mathrm{H}$, et al. Augmenting mental health in primary care: a 1-year study of deploying smartphone apps in a multi-site primary care/behavioral health integration program. Front Psychiatry. 2019 Feb;10:94. https://doi.org/10.3389/fpsyt.2019.00094

16. Lucas GM, Gratch J, King A, et al. It's only a computer: virtual humans increase willingness to disclose. Comput Human Behav. 2014 Aug;37:94-100. https://doi.org/10.1016/j.chb.2014.04.043

17. Morency L, Stratou G, DeVault D, et al. SimSensei demonstration: a perceptive virtual human interviewer for healthcare applications In: Proceedings of the Twenty-Ninth AAAI Conference on Artificial Intelligence and the Twenty-Seventh Innovative Applications of Artificial Intelligence Conference; 2015 Jan 25-30; Austin, TX. Palo Alto (CA): AAAI Press; 2015. p. 4307-8.

18. Carleton RN, Collimore KC, McCabe RE, et al. Addressing revisions to the Brief Fear of Negative Evaluation scale: measuring fear of negative evaluation across anxiety and mood disorders. J Anxiety Disord. 2011 Aug;25(6):822-8. https://doi.org/10.1016/j.janxdis.2011.04.002

19. van der Heijden PG, van Gils G, Bouts J, et al. A comparison of randomized response, computer-assisted self-interview, and faceto-face direct questioning. Sociol Methods Res. 2000;28(4):505-37. https://doi.org/10.1177/0049124100028004005

20. Choudhury M De, Kiciman E, Dredze M, et al. Discovering shifts to suicide ideation from mental health content in social media. In: CHI '16: Proceedings of the 2016 CHI Conference on Human Factors in Computing Systems; 2016 May 7-12; San Jose, CA. New York (NY): Association for Computing Machinery; 2016. p. 2098-110. https://doi. org $/ 10.1145 / 2858036.2858207$

21. Reger GM, McClure ML, Ruskin D, et al. Integrating predictive modeling into mental health care: an example in suicide prevention. Psychiatr Serv. 2019 Jan;70(1):71-4. https://doi.org/10.1176/appi. ps.201800242

22. Barrett PM, Steinhubl SR, Muse ED, et al. Digitising the mind. Lancet. 2017 May;389(10082):1877. https://doi.org/10.1016/S01406736(17)31218-7

23. Williams D, Hutton H, Ryan G. Creating value in healthcare: the need for innovative solutions. Healthc Pap. 2017;16(3):47-51. https://doi. org/10.12927/hcpap.2017.25080

24. Rauws M. X2AI executive summary [Internet]. X2AI; 2019 May 14 [updated 2020 Jan 6; cited 2020 Jan 21]. Available from: https://www. x2ai.com/blog/x2-executive-summary

25. Zhou T, Li X, Pei Y, et al. Internet-based cognitive behavioural therapy for subthreshold depression: a systematic review and meta-analysis. BMC Psychiatry. 2016;16:356. https://doi.org/10.1186/s12888-016-1061$\underline{9}$

26. Dunn AG, Mandl KD, Coiera E, et al. Social media interventions for precision publich health:promises and risks. NPJ Digit Med. 2018;1:47. https://doi.org/10.1038/s41746-018-0054-0

27. Olhede SC, Wolfe PJ. The growing ubiquity of algorithms in society: Implications, impacts and innovations. Philos Trans R Soc A. 2018;376(2128):20170364. https://doi.org/10.1098/rsta.2017.0364

28. Mulder T, Jagesar RR, Klingenberg AM, et al. New European privacy regulation: assessing the impact for digital medicine innovations. Eur Psychiatry. 2018 Oct;54:57-8. https://doi.org/10.1016/j. eurpsy.2018.07.003

29. Smetanin P, Stiff D, Briante C, et al. The life and economic impact of major mental illnesses in Canada: 2011 to 2041. Toronto, ON: RiskAnalytica, on behalf of the Mental Health Commission of Canada; 2011. 199 p. 